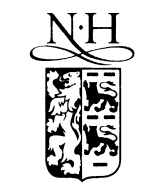

\title{
SIMS and XPS characterization of CdS/CdTe heterostructures grown by $\mathrm{MBE}$
}

\author{
P. Boieriu ${ }^{\text {a }}$, R. Sporken ${ }^{\text {a,b }}$, A. Adriaens ${ }^{\text {c,* }}$, Yan Xin ${ }^{\text {a }, ~ N . D . ~ B r o w n i n g ~ a, ~}$ \\ S. Sivananthan ${ }^{\text {a }}$ \\ a Microphysics Laboratory, Department of Physics (M/C 273), University of Illinois at Chicago, 845 W. Taylor Street, Chicago, \\ IL 60607, USA \\ b Laboratoire Interdisciplinaire de Spectroscopie Electronique, Facultés Universitaires Notre-Dame de la Paix, Rue de Bruxelles 61,5000 \\ Namur, Belgium \\ ${ }^{\mathrm{c}}$ Department of Chemistry, University of Antwerp, Universiteitsplein 1, 2610 Antwerp, Belgium
}

\begin{abstract}
In this study we have grown epitaxial layers of wurtzite-CdS on $\operatorname{CdTe}(\overline{1} \overline{1} \overline{1}) \mathrm{B} / \mathrm{Si}$ substrates using molecular beam epitaxy. Indium was used to obtain n-type doping of CdS. The concentration and uniformity of In was determined by secondary ion mass spectrometry (SIMS). Indium profiles were obtained for concentrations ranging from $5 \times 10^{17}$ to $1.4 \times 10^{21} \mathrm{~cm}^{-3}$ and agree well with the variation expected from the In flux. @ $\odot 2000$ Elsevier Science B.V. All rights reserved.
\end{abstract}

PACS: 81.05.Dz; 81.15.Hi; 82.80.Ms

Keywords: Solar cells; CdS/CdTe; Molecular beam epitaxy; SIMS

\section{Introduction}

Cadmium sulfide is a wide band gap semiconductor with a forbidden energy gap $E_{\mathrm{g}}$ of $2.42 \mathrm{eV}$. It is widely used to make inexpensive thin-film solar cells based on CdS/CdTe heterojunctions. $\mathrm{CdS}$ can be doped n-type whereas p-type is very difficult due to strong self-compensation. Both nand p-type CdTe can be obtained by doping it with In and As, respectively [1,2]. A natural choice is

\footnotetext{
${ }^{*}$ Corresponding author. Fax: +32-2-820-2376.

E-mail address: mieke@uia.ua.ac.be (A. Adriaens).
}

then to form the $\mathrm{p}-\mathrm{n}$ junction with a n-type $\mathrm{CdS}$ layer on top of a p-type CdTe layer. In this configuration, $\mathrm{CdS}$ is part of the $\mathrm{p}-\mathrm{n}$ junction but also acts as a window.

Molecular beam epitaxy (MBE) is well-known for its capability to grow semiconductor heterostructures with excellent structural quality. Few attempts to grow $\mathrm{CdS}$ with the metastable zincblende structure by MBE have been reported in the literature [3,4]. Recently, we have shown that MBE can be used to grow the thermodynamically stable wurtzite phase of CdS on CdTe $\left(\begin{array}{lll}\overline{1} & \overline{1} & \overline{1}\end{array}\right) \mathrm{B}$ [5].

The present paper discusses both the growth parameters and a multianalytical approach. Using 
reflection high-energy electron diffraction (RHEED), X-ray photoelectron spectroscopy (XPS) and secondary ion mass spectrometry (SIMS) to characterise the CdS/CdTe structures.

\section{Experiment}

CdS epilayers were grown on single crystalline CdTe ( $\left.\begin{array}{lll}\overline{1} & \overline{1} & \overline{1}\end{array}\right) \mathrm{B} / \mathrm{Si}\left(\begin{array}{lll}1 & 0 & 0\end{array}\right)$ or CdTe $\left(\begin{array}{lll}\overline{1} & \overline{1} & \overline{1}\end{array}\right) \mathrm{B} / \mathrm{Si}\left(\begin{array}{lll}1 & 1 & 1\end{array}\right)$ substrates. For the growth we used a Knudsen effusion cell providing the $\mathrm{Cd}$ flux and a valved $\mathrm{S}$ cracker cell with a source temperature of 130 $135^{\circ} \mathrm{C}$ and a cracking zone of $500^{\circ} \mathrm{C}$. A conventional effusion cell has been used as In source for n-type doping. The $\mathrm{Cd}$ cell temperature was maintained at $175^{\circ} \mathrm{C}$. The substrate temperature was between 200 and $300^{\circ} \mathrm{C}$. More details about the sample preparation have been described elsewhere [5].

XPS spectra were recorded with an SSX-100 spectrometer using a monochromatic and focused Al $\mathrm{K}_{\alpha}$ source $(h v=1486.6 \mathrm{eV})$. The binding energies were measured with respect to the Fermi level. The XPS spectrometer is connected to the CdS growth chamber through a system of UHV transfer modules.

SIMS depth profiles were performed with a CAMECA IMS 4f ion microprobe (Cameca, France). The samples were sputtered using a $\mathrm{Cs}^{+}$ source with an impact energy of $5.5 \mathrm{keV}$ or 14.5 $\mathrm{keV}$ depending on whether positive or negative secondary ions were acquired. The primary beam intensity was $100 \mathrm{nA}$; the area of analysis a few $\mu \mathrm{m}^{2}$. Instrumental parameters were kept constant within one analysis day to be able to compare between different samples.

\section{Results and discussion}

Before growing CdS, a thin buffer layer of CdTe was grown (about $100 \mathrm{~nm}$ ) to obtain a smooth starting surface using a single CdTe conventional boron nitride effusion (growth rate of 0.8 $\AA / s$ ). Due to the background pressure of sulphur this leads to the growth of pseudomorphic $\mathrm{CdTe}_{1-x} \mathrm{~S}_{x}$ strained to match the in-plane lattice parameter of the CdTe layer [5]. This ternary layer has a wurtzite structure and contains many stacking faults. CdS then grows with its own relaxed lattice parameter.

RHEED was used to monitor the MBE growth of CdS. Results showed bright streaks with no additional spots for CdS epilayer thickness of up to several hundreds of nanometers. The surface has the same hexagonal symmetry as the CdTe buffer layer. High resolution electron microscopy and selected area electron diffraction show that the $\mathrm{CdS}$ grows with the wurtzite structure, which is the most stable form of CdS. The epitaxial relationship is CdS [0 100$] / / \mathrm{CdTe}\left[\begin{array}{lll}1 & 1 & \overline{2}\end{array}\right]$ and $\mathrm{CdS}\left[\begin{array}{lll}0 & 0 & 1\end{array}\right] / /$ CdTe $\left[\begin{array}{lll}1 & 1 & 1\end{array}\right]$. Above a certain thickness $(\sim 2500 \AA)$ the RHEED patterns become spotty due to surface roughening.

XPS has been used in order to determine the composition of the epilayers. Taking advantage of the high sensitivity of XPS to indium, we have used this technique to find out if large concentrations of In can be incorporated into the CdS layer during MBE growth. Also quantitative analysis is straightforward in the case of XPS compared to SIMS, where sensitivity factors are known to depend very strongly on matrix effects. Therefore, we used samples with high In concentration, where the concentration could be measured by XPS, as reference samples to determine the SIMS sensitivity factors to be used for samples with lower concentration. Although there is no guarantee that sensitivity factors will be constant over several orders of magnitude, this procedure should give a much better estimate of the In concentration from SIMS data than using tabulated sensitivity factors.

The atomic composition was determined by XPS according to the following equation:

$[\mathrm{In}]=\frac{I_{\mathrm{In}} / S_{\mathrm{In}}}{\sum_{i} I_{i} / S_{i}}$.

In this equation $I_{i}$ represents the measured intensity for element $I$, while $S_{i}=\sigma_{i} E^{0.7}$ is the XPS sensitivity factor, where $\sigma_{i}$ is the photoionisation cross-section calculated by Scofield and $E$ is the kinetic energy of the photoelectrons [6]. The exponential term takes the energy dependence of the photoelectron escape depth and of the analyser 
transmission into account. This calculation assumes that the samples have a uniform composition. In particular, surface segregation or depletion of In would lead to wrong results. However, SIMS and Auger depth profiles show that the depth distribution of In is homogeneous for the sample that was used for this calibration.

SIMS depth profiles were acquired to study the presence of impurities in the layers, possible interdiffusion phenomena between the layers and the homogeneity and concentration of the In doping. Fig. 1 shows a depth profile of sample 101315. Positive secondary ions were acquired and the Figure shows the signals for $\mathrm{Cd}, \mathrm{Te}, \mathrm{In}, \mathrm{S}, \mathrm{K}$ and $\mathrm{Na}$. The depth profile shows both CdS and CdTe layers without any significant interdiffusion. Near the $\mathrm{CdS} / \mathrm{CdTe}$ interface, an enrichment of $\mathrm{Na}$ and $\mathrm{Al}$ is observed, which is located within the CdTe buffer layer that was grown in the $\mathrm{CdS}$ growth chamber. This contamination was not seen in all the samples and may be due to the CdTe source material used for this particular growth run. Profiles were also acquired for negative secondary ions, for which signals of $\mathrm{Se}, \mathrm{As}, \mathrm{Cl}, \mathrm{C}, \mathrm{F}$ and $\mathrm{O}$ were detected. All of them are present at the surface of the CdS layer, while at the CdS/CdTe interface an enrichment of oxygen can be seen for most of the samples. Occasionally a $\mathrm{Cl}$ contamination was detected near the CdS/CdTe interface.

The indium concentration is homogeneously distributed in the CdS layer in samples 36410b and

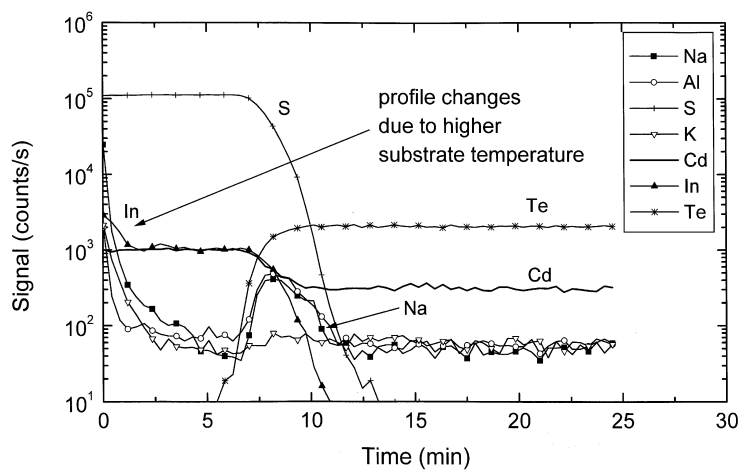

Fig. 1. SIMS depth profile of sample 101315. Signals for positive secondary ions of $\mathrm{Cd}, \mathrm{Te}, \mathrm{S}, \mathrm{In}, \mathrm{Na}, \mathrm{Al}$ and $\mathrm{K}$ are shown as a function of sputter time.

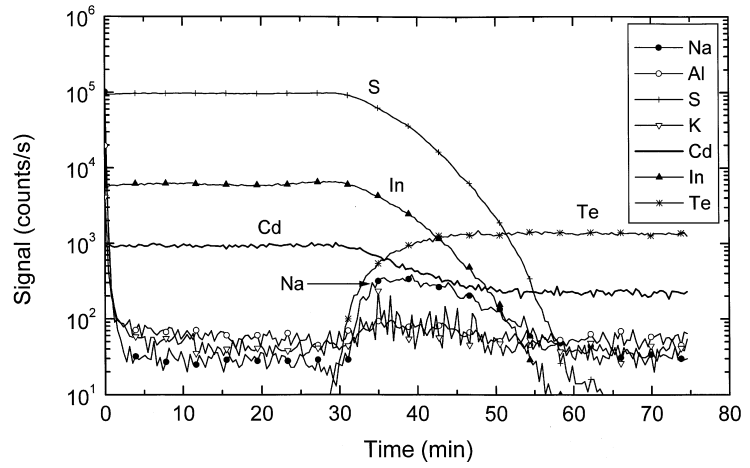

Fig. 2. SIMS depth profile of sample 41811. Signals for positive secondary ions of $\mathrm{Cd}, \mathrm{Te}, \mathrm{S}, \mathrm{In}, \mathrm{Na}, \mathrm{Al}$ and $\mathrm{K}$ are shown as a function of sputter time. Note that the scale in the $x$-axis differs from Fig. 1.

41811. As an example this is shown in Fig. 2 for sample 41811. For other samples, non-uniform In profiles have been observed and will be discussed below. The In concentration was calculated using relative sensitivity factors (RSF) and is shown in Fig. 3. The RSF for In was calculated using the XPS data of sample $36410 \mathrm{~b}$. The In concentration was then obtained according to:

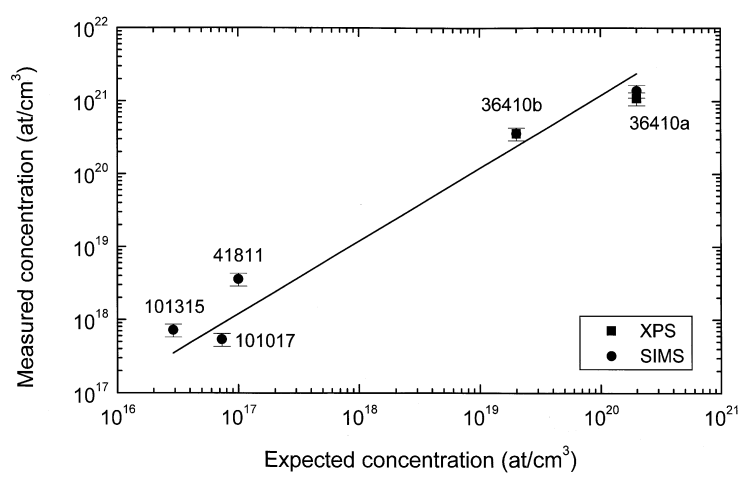

Fig. 3. Comparison between In concentrations measured by XPS, by SIMS and expected based on the calculated In flux. For XPS, Scofield photoionisation cross-sections were used. For SIMS, the In concentration deduced from XPS for sample $36410 \mathrm{~b}$ was used as a reference to calculate relative sensitivity factors. The expected In concentration is based on the calculated In/Cd flux ratio, assuming constant In sticking coefficient, but taking the variation of growth rate with substrate temperature into account. The solid line corresponds to the expected In concentration (multiplied by a factor of 10 ). 
$[\mathrm{In}]=\frac{I_{\mathrm{In}}}{I_{\mathrm{Cd}}} \cdot \mathrm{RSF}$

where [In] is the concentration of In, while $I_{\text {In }}$ and $I_{\mathrm{Cd}}$ are the measured SIMS intensities of In and $\mathrm{Cd}$. The results are compared with the expected concentration based on the calculated In flux, assuming identical values for the sticking coefficient for In and $\mathrm{Cd}$ at the lowest substrate temperature $\left(200^{\circ} \mathrm{C}\right)$. Samples 101017 and 101315 were grown at $300^{\circ} \mathrm{C}$ and therefore the growth rate was lower as is shown in Table 1. For the calculation of the expected In concentration, we assumed that the In sticking coefficient does not change with temperature in the range used here, whereas the change of the Cd sticking coefficient with substrate temperature was taken into account. The experimental values are in good agreement with the relative values of the In concentration over more than three decades, but the absolute values are about an order of magnitude higher than the expected ones. This difference can be attributed to the difficulty to calculate the $\mathrm{Cd}$ and In flux at the substrate. Also the absolute value of the $\mathrm{Cd}$ sticking coefficient is not known. However, from the good agreement between relative measured values and expected ones (taking variation of growth rate with substrate temperature into account), we can conclude that the In concentration depends on the substrate temperature used for the CdS growth via a variation of the growth rate (see Table 1).

The In enrichment at the surface of sample 101315 can then be explained by the dependence

Table 1

Substrate temperature, growth rate and In concentration for each of the samples, all listed for the main part of the layer. Sometimes different conditions were used at the beginning and at the end of the growth

\begin{tabular}{rlll}
\hline Sample & $\begin{array}{l}\text { Substrate } \\
\text { Tempera- } \\
\text { ture }\left({ }^{\circ} \mathrm{C}\right)\end{array}$ & $\begin{array}{l}\text { Growth } \\
\text { rate } \\
\left(\AA \mathrm{A} \mathrm{s}^{-1}\right)\end{array}$ & $\begin{array}{l}\text { In concentrationas } \\
\text { determined by } \\
\text { SIMS }\left(\text { at. cm }{ }^{-3}\right)\end{array}$ \\
\hline $36410 \mathrm{a}$ & 200 & 3.2 & $1.4 \times 10^{21}$ \\
$36104 \mathrm{~b}$ & 200 & 3.2 & $3.6 \times 10^{20}$ \\
41811 & 300 & 1.6 & $3.6 \times 10^{18}$ \\
101017 & 300 & 1.6 & $5.4 \times 10^{17}$ \\
101315 & 300 & 1.6 & $7.2 \times 10^{17}$ \\
\hline
\end{tabular}

on the substrate temperature. Indeed, the growth was stopped for a while to record RHEED patterns and a thin layer of $\operatorname{CdS}(200 \AA)$ was then grown at higher substrate temperature, but with the same In flux. Similar dependence of In concentration on substrate temperature was observed for other samples.

In and $\mathrm{Au}$ contacts were then evaporated on some of the In-doped CdS layers and $I-V$ characteristics measured between pairs of In and $\mathrm{Au}$ contacts. For the sample with low In concentration (sample 101315, $[\mathrm{In}]=5.4 \times 10^{17} \mathrm{~cm}^{-3}$ ), strong rectifying behaviour is observed, suggesting that a Schottky-type contact was formed between $\mathrm{Au}$ and CdS, since it is known that In forms ohmic contacts on n-type CdS.

\section{Conclusions}

CdS was grown by MBE on single crystalline CdTe $\left(\begin{array}{ll}1 & 1 \\ 1\end{array}\right) \mathrm{B} / \mathrm{Si}$ substrates. The CdS layers have a wurtzite structure and are epitaxial on CdTe. In was used to dope the CdS layers n-type. SIMS has shown that In concentrations between $5 \times 10^{17}$ $\mathrm{cm}^{-3}$ and $1.4 \times 10^{21} \mathrm{~cm}^{-3}$ are obtained. The measured values of the In concentration are in good agreement with the variation expected based on the In flux. Moreover, it is found that the In concentration depends on the substrate temperature. The experimental results suggest that the In incorporation does not depend on substrate temperature in the range investigated here $(200$ $300^{\circ} \mathrm{C}$ ), but $\mathrm{Cd}$ incorporation and hence the growth rate, changes by about a factor 2 . Homogeneous In concentration can then be obtained if the growth conditions and in particular the temperature are well controlled.

\section{Acknowledgements}

We thank F. Marchal for the Auger depth profile. R.S. acknowledges support from the Belgian National Fund for Scientific Research (FNRS). A.A. is indebted to the Fund of Scientific Research - Flanders (FWO). This work was 
supported in part by the Belgian Office for Scientific, Technical and Cultural Affairs (PAI 4/10).

\section{References}

[1] F. Bassani, S. Tatarenko, K. Saminadayar, J. Bleuse, N. Magnea, L.L. Pautrat, Appl. Phys. Lett. 58 (1991) 2651.
[2] J.M. Arias, S.H. Shin, D.E. Cooper, M. Zandian, J.G. Pasko, E.R. Gertner, R.E. DeWames, J. Vac. Sci. Technol. A 8 (1990) 1125.

[3] W.G. Wilke, R. Seedorf, K. Horn, J. Vac. Sci. Technol. B 7 (1989) 807.

[4] D.W. Niles, H. Höchst, Phys. Rev. B 41 (1990) 12710.

[5] P. Boieriu, R. Sporken, Y. Xin, N.D. Browning, S. Sivananthan, J. Electron Mat., in press.

[6] J.H. Scofield, J. Electron Spectrosc. 8 (1976) 129. 